\title{
Vulnerabilidad socioeconómica al incremento del nivel del mar en Tampico, Tamaulipas
}

\section{Socioeconomic vulnerability to sea level rise in Tampico, Tamaulipas}

\author{
Tovar Cabañas, Rodrigo; Jáuregui Díaz, José Alfredo; Vargas \\ Castilleja, Rocío del Carmen; Rolón Aguilar, Julio Cesar; Berrios- \\ Zepeda, Editor Academico Prof. Dr. Roberto Arturo
}

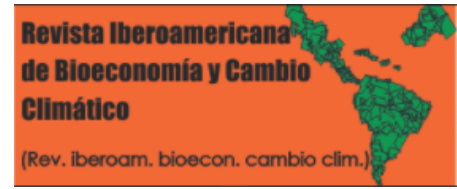

Rodrigo Tovar Cabañas rodrigo.tovarc@uanl.mx

(1) Universidad Autónoma de Nuevo León, Instituto de Investigaciones Sociales. , México

(iD José Alfredo Jáuregui Díaz jose.jaureguid@uanl.mx

Universidad Autónoma de Nuevo León, Instituto de Investigaciones Sociales. , México

DD Rocío del Carmen Vargas Castilleja rocvargas@docentes.uay.edu.mx Universidad Autónoma de Tamaulipas, Facultad de Ingeniería Arturo Narro Siller, Ave. Universidad y Blvd. Adolfo López Mateos, Centro Universitario Tampico-Madero, México

(iD) Julio Cesar Rolón Aguilar jrolon@docentes.uat.edu.mx Universidad Autónoma de Tamaulipas, Facultad de Ingeniería Arturo Narro Siller, Ave. Universidad y Blvd. Adolfo López Mateos, Centro Universitario Tampico-Madero, México

Editor Academico Prof. Dr. Roberto Arturo BerriosZepeda

Universidad Nacional Autónoma de Nicaragua, Leon. Facultad de Ciencias Económicas y Empresariales., Nicaragua

Revista Iberoamericana de Bioeconomía y Cambio Climático

Universidad Nacional Autónoma de Nicaragua, León, Nicaragua ISSN-e: 2410-7980

Periodicidad: Semestral

vol. 4, núm. 8, 2018

czuniga@ev.unanleon.edu.ni

Recepción: 06 Septiembre 2018

Aprobación: 13 Noviembre 2018

URL: http://portal.amelica.org/ameli, jatsRepo/394/3941755012/index.html
Resumen: Los estudios de la vulnerabilidad en costas mexicanas es un tema emergente ante el riesgo inminente por los efectos del cambio climático, impactando a los asentamientos humanos, el turismo, las actividades económicas, los ecosistemas costeros, entre otras. La importancia de la investigación radica en que cada vez más, la incertidumbre del cambio climático, aunada al incremento de desastres, repercute en el desarrollo endógeno de las localidades costeras de México y América Latina. Por lo que esta investigación, tiene como objetivo disertar los impactos económicos, sociales y culturales que puede sufrir el puerto de Tampico, Tamaulipas, bajo el escenario de un incremento súbito en el nivel del mar de 5 metros. El proceso metodológico consistió en geografizar los grupos vulnerables que se encuentran actualmente dentro de la zona de peligro. Es de advertir que en el tratamiento cuantitativo requirió del manejo de microdatos, mientras que las técnicas cualitativas, aplicadas durante el trabajo de campo, representaron un enfoque equilibrado interdisciplinario. Dentro de los resultados más apremiantes se destaca que, si el municipio de Tampico sufriera una inundación de cinco metros sobre el nivel del mar, $58 \mathrm{~km} 2$ quedarían anegados (63\% del total territorial), los cuales representan un valor superior a 7 mil millones de dólares. Con base en lo anterior, se requiere de la formulación de políticas dirigidas a grupos vulnerables específicos para reducir la sensibilidad de los sistemas y desarrollar ciudades costeras resilientes.

Palabras clave: Geografía Litoral, Vulnerabilidad Sociocultural, Impacto Económico.

Abstract: Studies of vulnerability on Mexican coasts is an emerging issue in the face of imminent risk from the effects of climate change, impacting human settlements, tourism, economic activities, coastal ecosystems, among others. The importance of the research is that, increasingly, the uncertainty of climate change, coupled with the increase in disasters, has an impact on the endogenous development of the coastal localities of Mexico and Latin America. So this research, aims to discuss the economic, social and cultural aspects that may occur in the port of Tampico, Tamaulipas, under the scenario of a sudden increase in sea level of 5 meters. The methodological process consisted in geographizing the vulnerable groups that are currently within the danger zone. It should be noted that in the 
DOI: https://doi.org/10.5377/ribcc.v4i8.6714

Autor de correspondencia: rodrigo.tovarc@uanl.mx quantitative treatment required the management of micro-data, while qualitative techniques, applied during fieldwork, represent a balanced interdisciplinary approach. Among the most pressing results is that, if the municipality of Tampico suffered a flood of five meters above sea level, $58 \mathrm{~km} 2$ would be flooded $(63 \%$ of the total territorial), which represented a value exceeding 7 billion Dollars. Based on the above, it is necessary to formulate policies aimed at vulnerable groups in order to reduce the sensitivity of the systems and to develop resilient coastal cities.

Keywords: Coastal Geography, Sociocultural Vulnerability, Economic Impact.

\section{INTRODUCCIÓN}

Tras la inundación de 1955, uno de los primeros estudios tendentes a resolver el problema de inundaciones en Tampico fue propuesto por López (1961), en tanto que el primer mapa de zonas de inundación fue elaborado en la década de los años ochenta del siglo XX por (Zamora Ramirez, 1990). A comienzos de este siglo Jiménez (2007) expone, de manera breve, los riesgos de inundación de la conurbación Tampico, Madero y Altamira. Según Jiménez, la conurbación se inunda debido a la fisiografía natural de la bahía y a una ausencia de planificación territorial, principalmente en varias zonas, tal como: Nuevo Madero, Santa Elena, Moralillo y Cascajal por un lado y riveras de la Laguna del Chairel. En García (2008) a partir de una serie de escenarios de inundación a diferentes intensidades de ciclones tropicales determinó que alrededor de 7 colonias, principalmente del sur de Ciudad Madero eran las más vulnerables, dejando pendiente el caso del municipio de Tampico. Colateralmente existen una serie de mapas elaborados por Vázquez (2009) sobre la cobertura médica de la ciudad de Tampico, los cuales pueden ayudar a dimensionar la vulnerabilidad social de la misma.

Por su parte Lambarri (2010), desde una postura ecomarxista realiza por una parte una breve cronología hidráulica, ambiental, dando énfasis a las trayectorias de los huracanes acaecidos en Tampico, y por otra un análisis geoestadístico para sugerir un ordenamiento territorial y ecológico, que impida más asentamientos en zonas de riesgo, de lo contrario, señala, que los 80 mil habitantes que viven en la zona de riesgo podrían aumentar exponencialmente su número. De manera parecida, Sánchez (2011), mediante un análisis espacial de las contingencias ambientales o hidrológicas y de las trayectorias históricas de los ciclones tropicales ocurridos en Tampico durante los últimos 70 años, argumenta reiterativamente, que más de 160 mil habitantes están en riesgo y que existe un 35\% de probabilidades de que en el transcurso de la década sufran el embate de un huracán categoría 1.

Desde el ámbito de las ciencias de la comunicación Rosas (2012), advierte que en Tampico pese a sus múltiples experiencias con desastres naturales aún no se ha consolidado una cultura de prevención de desastres efectiva, con códigos, lenguajes y valores capaces de mantener los hábitos de la sobrevivencia humana, la cual al parecer se ha perdido, pues el número de personas en riesgo año con año va en aumento. Además, al riesgo de inundaciones habría que agregar otros problemas o riesgos que se están manifestando en Tampico, tal es el caso de las islas de calor Fuentes (2015), la erosión del litoral, la intención de trasvasar agua a otras ciudades del norte en época de estiaje, lo que implicaría una alteración en la disponibilidad del

\section{NotAS DE AUTOR}


recurso Sánchez et al. (2014), el incremento de los desechos industriales y la tergiversación de los problemas por parte de los medios de comunicación masiva (Millán, Tello, \& Gómez, 2016).

Con base en Campos (2012), es probable que la falta de cultura de prevención se deba a lo que observa en torno a las predicciones anuales sobre seguridad hidrológica y al escás de registros pluviográficos, lo que origina falsos patrones o correlaciones imprecisas, como el hecho de asociar intensidades o rutas de huracanes con procesos de vulnerabilidad. Es decir, conforme a Campos hace falta ampliar la observación pluviométrica hacia las partes altas de las cuencas involucradas con el puerto de Tampico. Tampoco habría que perder de vista que la predicción del incremento del nivel del mar en el sur de Tamaulipas, es compleja e incierta, existe un estudio sobre el comportamiento de las elevaciones del nivel del mar en la costa hacia el año 2050 para algunos puntos específicos del estado destacando la zona sur con mayores elevaciones, sin embargo, este nivel varía anualmente durante la temporada de inundaciones, lo que muestra la complejidad del sistema (Ortega, 2013).

Es conveniente señalar que la característica principal de esos y otros trabajos es el uso indiscriminado de las escalas cartográficas que se emplean en el análisis de la vulnerabilidad, este inconveniente conduce a una inconsistencia en la sobreposición de los factores físicos y sociales, de modo que sus coropletas, si bien es cierto que pueden ser empleadas para la predicción de escenarios de riesgo por fenómenos hidrometeorológicos, de gran valía para la concientización de la población sobre la peligrosidad de determinados fenómenos naturales, pero les hace falta una mejor yuxtaposición tendente a resolver los cómo de la planificación y el desarrollo sustentable que exige nuestra actual coyuntura en materia de cambio climático.

Por ejemplo, a más de medio siglo de la gran inundación que sufrió Tampico, en donde se dejó notar cómo el incremento del nivel del mar es la variable independiente, el Puerto de Tampico sólo cuenta con el atlas de riesgos hidrometeorológicos SENAPRED Tamaulipas (2009) y un Programa Estatal de Cambio Climático Secretaría de Desarrollo Urbano y Medio Ambiente - SEDUMA (2016) , a escala estatal y municipal. Por lo tanto, aún siguen sin resolverse ciertas preguntas como: ¿De qué tamaño es el área urbana a la que el mar le podría ganar a la tierra del municipio de Tampico, de incrementarse el nivel a 5 metros? ¿Cuáles son las principales construcciones en riesgo si el nivel del mar sube 5 metros en las próximas décadas?

¿Qué características socioeconómicas presenta la población que está en la zona cero o de riesgo ante un escenario como este? Por lo tanto, la presente investigación busca dar respuesta a esas y otras interrogantes que son pertinentes dada la complejidad actual de un sistema que está en constante crecimiento, cuyos riesgos se potencializan de no contar con herramientas sólidas de planeación y análisis para la toma de decisiones tendientes a la prevención y adaptación de las zonas más sensibles.

\section{INCREMENTO DEL NIVEL DEL MAR}

Estimar el nivel medio del mar es complicado ya que los mareógrafos registran variaciones considerables debido a que algunas áreas de tierra se levantan y otras se hunden, por lo que los cambios del nivel del mar todavía no son concluyentes. Sin embargo, en estudios recientes se ha observado que luego del Dryas Reciente, el mar subió a una tasa de $4 \mathrm{~m}$ por siglo Webster et al. (2004), en términos antropológicos dicha tasa es de $0.5 \mathrm{~mm} /$ año para el final del epipaleolítico y de $0.2 \mathrm{~mm} /$ año para los últimos 3 milenios Intergovernmental Panel on Climate Change - IPCC (2001), actualmente en Ámsterdam, el ascenso se estima en 1.5 mm/año. Por lo que a fin de siglo, se espera que el aumento del nivel del mar no sea uniforme en las distintas regiones del mundo, es decir, su impacto será muy variable de región a región.

Las evaluaciones científicas del IPCC (2007: 323) consideran un ascenso del nivel del mar de entre 19 a 58 $\mathrm{cm}$ hacia 2100; otros cálculos pronostican un ascenso de 0.9 a 1.3 metros para el mismo periodo (Grinsted, Moore, \& Jevrejeva, 2004). Sin embargo, no se pueden descartar las variaciones regionales provocadas por fenómenos hidrometeorológicos extremos, entre otros de orden astronómico. Por tal razón algunos científicos han elaborado modelos de costas anegadas hasta las cotas actuales de 10 y $5 \mathrm{msnm}$, como Cruz 
(2016: 115), quien a partir de un índice de vulnerabilidad costera ubica al municipio de Tampico como uno de los dos más vulnerables de todo el estado de Tamaulipas. Bajo estas consideraciones, se presentan escenarios para la cota $5 \mathrm{~m}$. de anegación del municipio de Tampico.

\section{TAMPICO A CINCO METROS BAJO LAS AGUAS DEL MAR}

A penas hace ocho años que en México el gobierno federal impulsó una iniciativa en materia de cambio climático, por lo que es de comprender que los escenarios y estimaciones a nivel local aún son escasos, por ejemplo, en 2010, la Universidad Autónoma Metropolitana, el Instituto de Ciencias del Mar y Limnología de la UNAM y la Universidad de Campeche hicieron el esfuerzo para ilustrar las tendencias y dinámicas del comportamiento del nivel del mar, gracias a eso se sabe que frente a Tampico el nivel del mar, en promedio, subió su nivel $15 \mathrm{~cm}$ de 1964 a 1979, posteriormente, aunque la tasa ha incrementado, se asume que en dicho puerto el nivel asciende $9.2 \mathrm{~cm}$ por década (Zavala, De Buen, \& Romero, 2011:325). A enriquecer parte de estas indagaciones ligadas al desarrollo local sustentable va dedicado este apartado.

Luego de aprovechar un método de fotogrametría satelital para obtener las curvas de nivel ${ }^{1}$ que sirvieron para ubicar y delimitar las zonas del territorio del municipio de Tampico que quedarían bajo el agua de mar, en caso de que éste subiera $5 \mathrm{~m}$ su nivel en las próximas décadas, se llegó a los resultados siguientes: de los $92.7 \mathrm{~km} 2$ con los que cuenta el municipio de Tampico Instituto Nacional de Estadística y Geografía - INEGI (2000), $58.3 \mathrm{~km} 2$ quedan por debajo de la cota de $5 \mathrm{msnm}$, lo que representa el 62.9\% del total municipal. Tomando en cuenta únicamente el área urbana total del municipio (aproximadamente 3,500 ha.), 4.4\% de su infraestructura urbana queda por debajo de la cota de $5 \mathrm{msnm}$.

La mayor parte de esas 443 hectáreas urbanizadas ${ }^{2}$, que desde ya están en riesgo de inundación en el Puerto de Tampico, Tamaulipas (Figura 1), corresponden al histórico Canal de la Cortadura y localidades circundantes, sobre las cuales vamos a investigar los principales impactos socioculturales y económicos que se podrían derivar a raíz de una subida del nivel del mar de cinco metros durante algún fenómeno hidrometeorológico extremo bajo el contexto de cambio climático. 


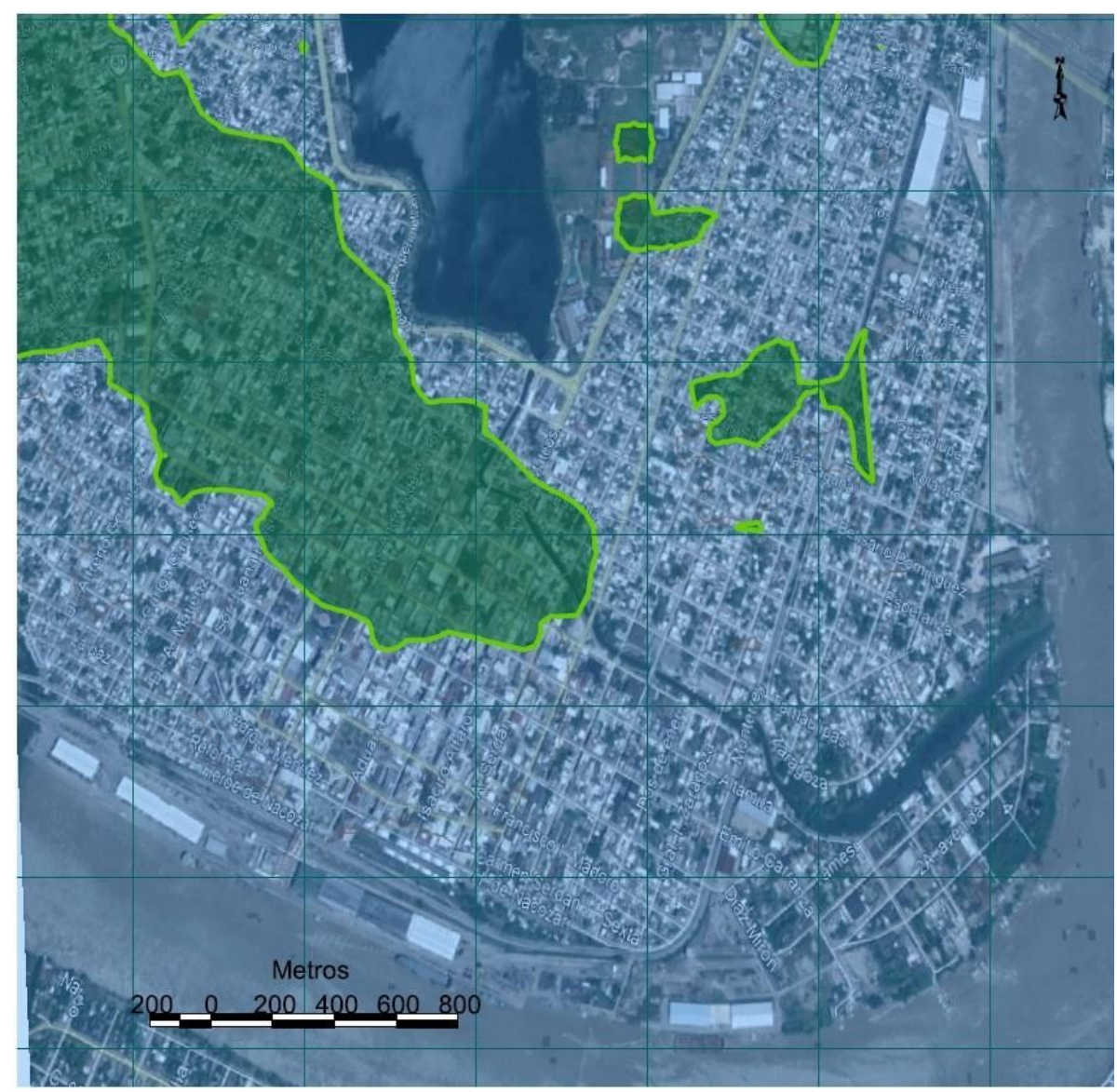

FIGURA 1.

Principales zonas bajas del municipio de Tampico.

Cálculos de los autores con base en datos proporcionados por Google Earth -SIO, NOAA, US Navy, NGA y GEBCO-.

\section{IMPACTOS ECONÓMICOS ANTE EL ESCENARIO DE INCREMENTO DEL MAR}

Una de las afectaciones principales del cambio climático en las zonas costeras de México se relaciona con el incremento del nivel del mar, la cual, económicamente, se puede estimar a partir de la cuantificación del Producto Interno Bruto (PIB) municipal o por la estimación del valor de los servicios ambientales que aportan ciertos ecosistemas. En ese sentido, en este apartado, vamos a reflexionar en torno a las cifras económicas del municipio de Tampico.

Conforme a González y Gallegos (2014), la distribución porcentual del PIB del año 2010, de los municipios de Tamaulipas, por región es el siguiente: Región Fronteriza 51.7\%; Valle de San Fernando 1.4\%; Centro 12.3\%; Región Sur 29.3\%; Mante 4.4\%; y Altiplano 0.9\%. Si consideramos el PIB estatal ${ }^{3}$ que arroja INEGI (2012), la Región Sur, a la que pertenece el municipio de Tampico, generó en total \$113,068 millones de pesos, de los cuales 52,949.65 millones los generó el municipio bajo estudio.

Cabe destacar que desde el punto de vista histórico dicha riqueza municipal, en función de la población económicamente activa, ha corrido a cargo del sector de los servicios y del comercio al menudeo, con una participación porcentual de $43 \%$ y $37 \%$ respectivamente Peraza (2016), cuyo personal ocupado, para el año de 2014, se estima en poco más de 60,000 empleados dedicados a las actividades comerciales y de servicios, y otros 5,500 relacionados con las actividades manufactureras (INEGI, 2015). Sobre el sector primario, cabe recordar que en el Puerto de Tampico son las actividades informales y la pesca artesanal o rivereña las que 
emplean unos 9,000 trabajadores, por lo que el PIB municipal depende de alrededor de 75,000 trabajadores, de los cuales más 3,500 laboran dentro de las zonas de peligro por inundación.

Otro impacto económico a discurrir en el escenario de riesgo bajo análisis es la capacidad instalada, que como activo tiene un valor para la administración territorial del municipio de Tampico. Por ejemplo, la red que integra su sistema carretero, conformado principalmente por avenidas, bulevares y calles, según INEGI (2010), tiene aproximadamente 841,202 kilómetros lineales, de los cuales 211,672 de ellos quedarían anegados (25.1\%) si el nivel del mar sube de nivel en cinco metros. Ahora bien, si aceptamos el costo promedio ( 25 dólares) que cuesta un metro cuadrado de asfalto, así como el ancho promedio de las calles (8 metros) en riesgo de inundación, tenemos que los $1,693,373 \mathrm{~m}^{2}$ resultantes, representan un valor de $\$ 804.3$ millones de pesos, si a esto le sumamos el valor del resto de infraestructura pública (alumbrado, aceras, drenaje, agua potable, etcétera), la cifra supera los 3,217.4 millones de pesos, (equivalente al 6.0\% del PIB municipal de 2010).

Por otra parte, el valor de los predios urbanizados (1077.4 ha), en riesgo de inundarse con una crecida del mar de $5 \mathrm{~m}$, cotizado en precios del mercado corriente ${ }^{4}$ es de $\$ 43$ mil millones de pesos (unos 2 mil millones de dólares), esa cifra equivale a 0.81 veces el PIB del municipio de Tampico del año 2010. Considerando el valor de los bienes raíces, tomando como base $\$ 100,000$ pesos por cada $60 \mathrm{~m}^{3}$ de construcción, es decir, predios no mayores a $10 \mathrm{~m}^{2}$ y de una sola planta, habría que agregar otros 0.1 billones de pesos, o sea $203 \%$ del PIB municipal. En resumen, la anegación por debajo de $5 \mathrm{~m}$ de la infraestructura de Tampico, podría impactar al 38\% del PIB del estado de Tamaulipas, sin embargo, si se consideran los bienes inmuebles entre otras afectaciones, la estimación equivaldría al total ${ }^{5}$ de dicho PIB estatal. En otros términos, el impacto de los efectos del cambio climático sobre el municipio de Tampico equivale al saldo de la deuda bruta nacional (externa e interna) del sector público del año de 1987, la cual la CEFP (2012) estima en 211,686 millones de pesos. Ahora bien, además de los 3,500 trabajadores afectados directamente por el escenario de cambio climático expuesto, es pertinente ampliarse y describir los impactos sociales que los efectos de una crecida del nivel de mar de $5 \mathrm{~m}$ dejarían sobre el puerto de Tampico.

\section{IMPACTOS SOCIALES ANTE EL ESCENARIO DE INCREMENTO DEL MAR}

Sin restar importancia a las cifras económicas, debemos señalar que es la demanda de vivienda de interés social la que experimentará los mayores cambios debido a los impactos por fenómenos hidrometeorológicos extremos detonados por el cambio climático. Por ejemplo, adicionalmente a las 18,673 solicitudes de vivienda que en promedio requiere el municipio de Tampico INFONAVIT (2014), se tendrían que sumar otras 19,266 correspondientes a todas aquellas viviendas de dicha demarcación que se sitúan por debajo de la cota de $5 \mathrm{msnm}$, es decir, los impactos secundarios del cambio climático incrementarían un $103.1 \%$ las necesidades de vivienda del Puerto de Tampico para la reubicación de la población de la zona cero.

Por otra parte, de acuerdo con la Dirección de Registro Público de Monumentos y Zonas Arqueológicas del INAH (2017), de los siete sitios arqueológicos con que cuenta el municipio de Tampico, el sitio denominado Tierra Alta, ubicado al noroeste, se sitúa a $7 \mathrm{msnm}$, sin embargo, el área de ocupación arqueológica se extiende hasta las colonias Independencia y Tierra Alta, situadas a una altura de $5 \mathrm{msnm}$, dicha área de ocupación mesoamericana aún no ha sido estudiada mediante el uso de nuevas tecnologías, tal como el georadar, entre otras técnicas de geocronología que ayuden a conocer la historia ambiental de dicha riqueza cultural.

Ahora bien, en términos de vulnerabilidad, en una primera instancia se podría suponer que este riesgo medioambiental no distingue la condición social de los futuros refugiados ambientales, empero, es preciso señalar que la margen sur de la desembocadura del río Pánuco prácticamente es la más riesgosa, en particular las viviendas ubicadas en la porción occidental de la colonia Cascajal (Figura 1), esta demarcación vecinal es 
la que tiene la menor cantidad de viviendas en situación de vulnerabilidad respecto a una crecida de río o potamoweack, 40 viviendas conforme a (INEGI, 2010).

En segundo término está el occidente de la colonia Morelos, que si bien es cierto que su riesgo es ligeramente menor al del Cascajal, la cantidad de viviendas lo hacen sumamente más vulnerable, puesto que en ese lugar se concentran 3,026 viviendas en potamoweack, mientras que el este de dicha colonia tiene 2,683 viviendas en potamoweack. Debido a las elevadas tasas de densidad de población que por lo regular presentan las casas de autoconstrucción de la clase baja, y dado que son estas las que integran el mayor número de viviendas en alto riesgo de ser envestidas por algún fenómeno hidrometeorológico, en la Colonia Morelos, se estima que son alrededor de 18,991 las personas en situación de potamoweack. Esta cifra representa la cuarta parte de lo estimado por Lambarri (2010) o la octava parte calculada por Sánchez (2011).

Con un breve índice de vulnerabilidad social se pudo conocer de forma relativa el nivel socioeconómico de las Áreas Geoestadísticas Básicas (AGEB's) en situación de potamoweack del Puerto de Tampico. Al respecto, se puede advertir que de las 20 AGEB's en potamoweack, se resalta que la colonia el Cascajal presenta el índice de vulnerabilidad social más alto; le sigue tanto el norte de la colonia Vicente Guerrero, como el oeste de la colonia Guadalupe Mainero; el tercer puesto lo ocupan tanto la zona central de la colonia Morelos, así como la Isleta Pérez; de acuerdo con el índice de vulnerabilidad social tanto el oeste como el oriente de la colonia Morelos se encuentran en un nivel 4 de potamoweack, por último las colonias Obrera y Árbol Grande presentan un nivel 5 conforme al índice expuesto, lo que las sitúa como las zonas menos vulnerables dentro de ese conjunto (Figura 2). Al respecto, conforme a los recorridos que se realizaron en trabajo de campo se puede advertir que las consecuencias del cambio climático revelan que en ciertos sectores urbanos, principalmente del sur del Puerto de Tampico, la deuda social sigue incrementándose.

En otras zonas bajo estudio, como en las colonias José López Portillo y la Enrique Cárdenas González (ambas ubicadas al norte de Tampico) paradójicamente pese a que sus pobladores empíricamente han resentido el embate de fuertes huracanes en más de una ocasión, la mayoría de ellos (mayoritariamente trabajadores informales), durante una situación post-desastre (como el ocurrido durante la temporada de huracanes del Atlántico del 2008), nunca han tenido el interés de acudir a un centro de refugio o albergue. De hecho, más de la mitad de sus habitantes han descartado definitivamente la idea de cambiarse de residencia, mientras que poco menos del $20 \%$ si desea vivir en un lugar con mayor altura o seguro, mientras que el resto no sabe qué hacer. 


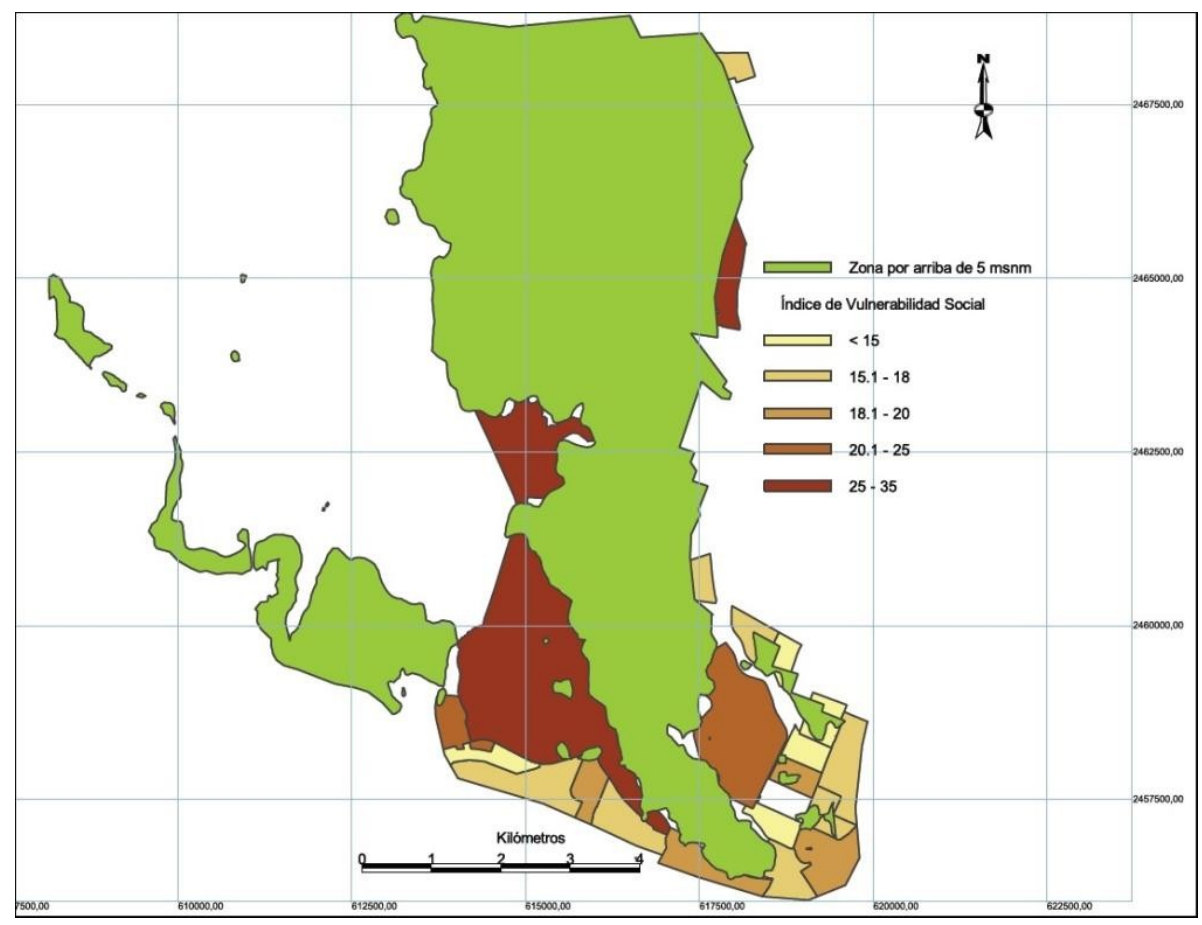

FIGURA 2.

Índice de Vulnerabilidad Social de las zonas por debajo de los $5 \mathrm{msnm}$ de Tampico, Tamaulipas. elaboración propia con base en (INEGI, 2011).

Se advierte que en este escenario tanto empresarios y gobiernos locales no han podido concretar una planificación urbana adecuada para afrontar y adaptarse a los fenómenos hidrometeorológicos extremos bajo contexto de cambio climático. Por tal motivo, la población ha decidido convivir con el peligro, pese a que comprometen su vida y patrimonio en ello (Figura 3). 


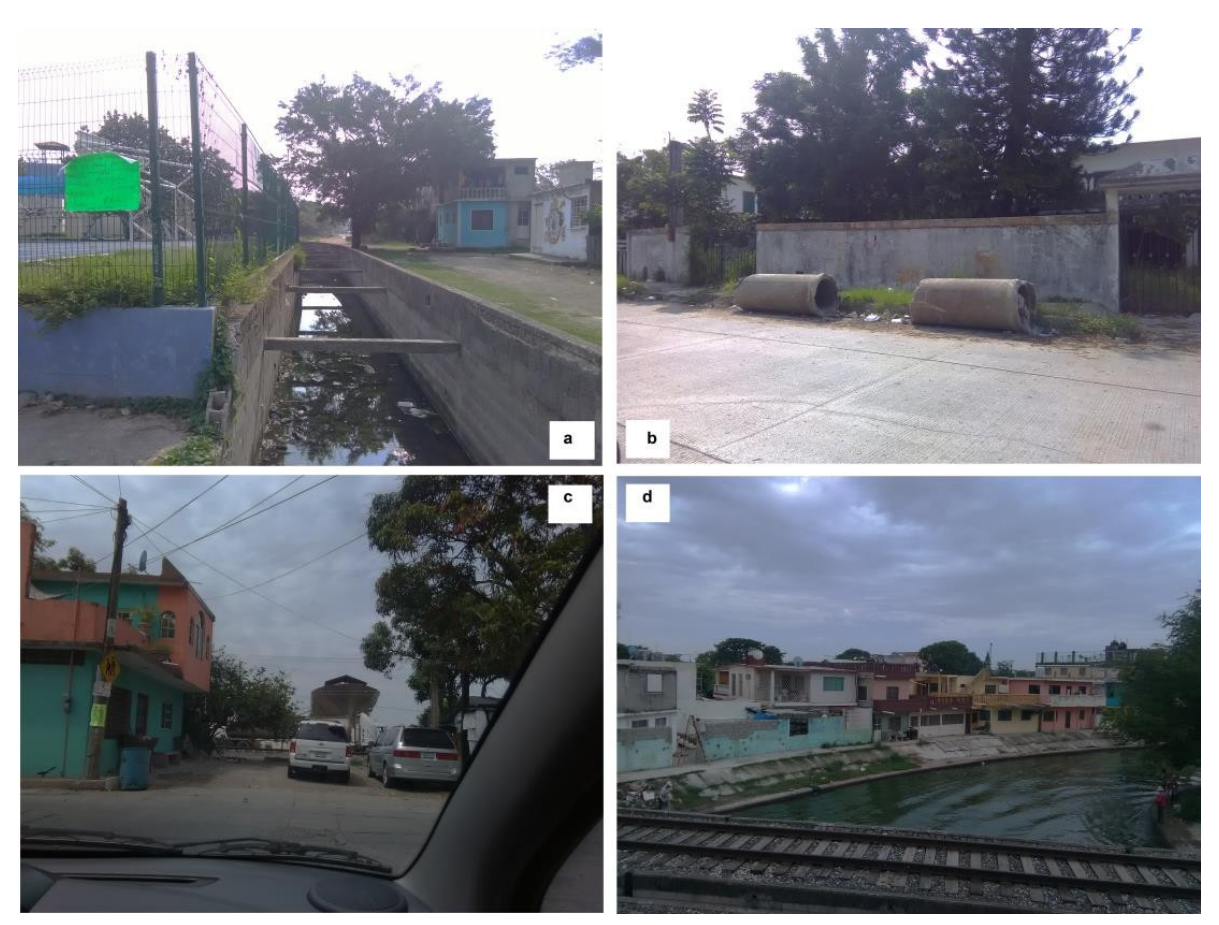

FIGURA 3.

Zonas bajas de la zona cero de Tampico: a) colonia Enrique Cárdenas González; b) colonia Tancol; c) colonia Morelos; d) colonia Guadalupe Victoria, Tampico, Tamaulipas, México, 21 de junio de 2018. elaboración propia con base en trabajo de campo, temporada 2017-2018.

\section{Conclusiones}

En el marco de las observaciones anteriores el tema del incremento del nivel mar en costas, no representa un valor sustancial a corto plazo, pero sí muestra el ritmo de crecimiento del riesgo, el cual se intensifica por la presencia de eventos hidrometeorológicos extremos, así como por la vulnerabilidad de los sectores más bajos del puerto, impactando en los costos para recuperarse, así como a la sociedad misma, que sí bien ha padecido inundaciones, empero, el arraigo por su territorio demerita el apoyo a la damnificación por este tipo de eventos que el gobierno local proporciona, y mantiene latente el riesgo, el cual puede traducirse en afectaciones a la salud, pérdidas de bienes inmuebles, entre otras. Tomando el escenario de un incremento del nivel del mar a la $5 \mathrm{~m}$. en el puerto, se estima que un 63\% de éste quedaría bajo situación de riesgo por inundación.

En consecuencia, la economía del puerto se vería afectada, disminuyendo el $47 \%$ de participación en el PIB estatal, afectando a $4.5 \%$ de personas ocupadas bajo alguna actividad productiva en la zona cero y valorando las pérdidas de la capacidad instalada en casi el 6\% del PIB municipal. Como resultado de los impactos sociales, se resalta la necesidad de incrementar la vivienda en más del 100\% para proteger a la población de la zona de análisis. Las colonias tales como Cascajal y Morelos, en el sur del puerto presentan un alto índice de vulnerabilidad social, debido a la concentración de población y de viviendas.

Es notable el costo de no planear las condiciones de urbanización considerando escenarios extremos, la identificación y valoración de las zonas de riesgos en ciudades vulnerables de México, son temas prioritarios en las agendas bajo las tres órdenes de gobierno, coadyuvando en los compromisos para mitigar y adaptarse a los efectos del cambio climático. 


\section{LiTERATURA CITADA}

Zamora Ramirez, I. (1990). Desarrollo urbano y nuevo asentamiento en la zona conurbada de Tampico cd. MaderoAltamira. (Tesis de pregrado) Universidad Nacional Autónoma de México, Facultad de Arquitectua. México: Universidad Nacional Autónoma de México.

Campos, D. (2012). Relación y estimación de predicciones de lluvia horaria-diaria en dos zonas geográficas de México. Tecnología y Ciencias del Agua, 3(2), 141-152.

CEFP. (2012). Indicadores y Estadísticas (Ingresos y Deuda): Costo Financiero de la Deuda Bruta del Sector Público Presupuestario, 1980-2012. México: Centro de Estudios de las Finanzas Públicas.

Cruz, C. (2016: 115). Evaluación de la Vulnerabilidad en las costas mexicanas. (Tesis de pregrado) Universidad Nacional Autónoma de México, Facultad de Ingeniería, Facultad de Ingeniería. México. México: Universidad Nacional Autónoma de México.

Fuentes, C. (2015). Islas de calor urbano en Tampico, México: Impacto del microclima a la calidad del hábitat. Nova scientia, 7(13), 495-515. https://doi.org/10.21640/ns.v7i13.41

García, R. (2008). Riesgo de inundación por marea de tormenta en el municipio de cd. Madero, Tamaulipas. (Tesis de pregrado) Universidad Autónoma de Tamaulipas, Facultad de Ingeniería “Arturo Narro Siller”. Tampico: Universidad Autónoma de Tamaulipas.

González, A., \& Gallegos, G. (2014). El producto interno bruto de los municipios de México: II. Estados MZ. Revista mexicana de ciencias agricolas, 5(8), 1405-1421.

Grinsted, A., Moore, J., \& Jevrejeva, S. (2004). Reconstructing sea level from paleo and projected temperatures 200 to 2100 ad. Climate Dynamics, 34(4), 461-472. https://doi.org/10.1007/s00382-008-0507-2

INEGI. (2000). Cuaderno estadístico municipal, Tampico, estado de Tamaulipas. Aguascalientes, México, México: Instituto Nacional de Estadística, Geografía e Informática.

INEGI. (2010). Cartografía geoestadística urbana, Cierre del Censo de Población y Vivienda 2010. Tampico. México: Instituto Nacional de Estadística, Geografía e Informática.

INEGI. (2011). Resultados definitivos: Censo de poblacio\#n y vivienda 2010. (G. e. Instituto Nacional de Estadística, Ed.) Aguascalientes, Aguascalientes, México: Instituto Nacional de Estadistica, Geografia e Informatica.

INEGI. (2012). Sistema de Cuentas Nacionales de México. Producto Interno Bruto por entidad federativa 2006-2010, Año base 2003. Segunda versión. México: Instituto Nacional de Estadística, Geografía e Informática.

INEGI. (2015). Censos económicos 2014. Aguascalientes : Instituto Nacional de Estadística y Geografía.

INFONAVIT. (2014). Demanda Potencial. ( $4^{\circ}$ bimestre del 2014). México, México, México: Instituto del Fondo Nacional de la Vivienda para los TrabajadoresInstituto, Nacional de Antropología e Historia, INAH. (2017). Catálogo de sitios arqueológicos Tampico. (I. N. Historia, Ed.) México, Ciudad de México, México.

Intergovernmental Panel on Climate Change, IPCC. (2001). Climate Change 2001: the scientific basis, Contribution of Working Group I to the Third Assessment Report of the Intergovernmental Panel on Climate Change. Cambridge: Cambridge University Press.

IPCC. (2007: 323). Climate Change 2007. Contribution of Working Group II to the Fourth Assessment Report of the Intergovernmental Panel on Climate Change. Cambridge: Cambridge University Press.

Jiménez, S. (2007). Los riesgos de inundación en la conurbación de Tampico-Madero-Altamira. CienciaUAT, 1(3), 48.

Lambarri, J. (2010). Construcción social de espacios riesgosos y desastres en Tampico. (Tesis de pregrado) Universidad Nacional Autónoma de México, Facultad de Filosofía y Letras. Ciudad de México: Universidad Nacional Autónoma de México.

López. (1961). Ante-proyecto preliminar encaminado a resolver el problema de inundaciones en Tampico y Ciudad Madero. Universidad Nacional Autónoma de México, Facultad de Ingeniería. México: Universidad Nacional Autónoma de México, Facultad de Ingeniería.

Millán, A., Tello, A., \& Gómez, A. (2016). Información ante escenarios de riesgos: caso Tampico, México. Derecom, 21, 63-80. 
Ortega, R. (2013). Evaluación y Monitoreo de la Vulnerabilidad al Cambio Climático de las Costas de Tamaulipas. Tampico: Centro de Investigación en Ciencia Aplicada y Tecnología Avanzada

Peraza, M. (2016). Plan Municipal de Desarrollo Tampico 2016 - 2018. Tampico: Administración municipal de Tampico 2016-2018.

Rosas, M. (2012). La responsabilidad social de los medios de comunicación masiva ante los riesgos de la zona conurbada de Tampico. Razón y Palabra, 16(80), 325-342.

Sánchez, D. (2011). Peligrosidad y exposición a los ciclones tropicales en ciudades del Golfo de México: El caso de Tampico. Revista de Geografia Norte Grande (50), 151-170. https://doi.org/10.4067/S071834022011000300009

Sánchez, G., Tobías, R., Vargas, R., \& Haces, M. (2014). Análisis preliminar del impacto del cambio climático en la disponibilidad de agua en la cuenca media del río Pánuco. En R. Tobías, G. Sánchez, J. Rolón, J. Treviño, \& E. Rolón, Cambio Climático en la zona noreste de México. Una introducción a temas de importancia regional. (págs. 27-41). México: Pearson.

Secretaría de Desarrollo Urbano y Medio Ambiente, SEDUMA. (2016). Programa Estatal de Cambio Climático 2015-2030. Cd. Victoria: Gobierno del Estado de Tamaulipas.

SENAPRED Tamaulipas. (2009). Atlas de Riesgos Tampico, Madero y Altamira. Cd. Victoria: Gobierno del Estado de Tamaulipas.

Vázquez, R. (2009). Estructura del sistema de salud en la conurbación de Tampico-Ciudad Madero-Altamira. (Tesis de posgrado) Universidad Nacional Autónoma de México, Facultad de Filosofía y Letras. México: Universidad Nacional Autónoma de México.

Webster, J., Clague, D., Riker-Coleman, K., Gallup, C., Braga , J., \& Cameron, D. (2004). Drowning of the -150 m reef off Hawaii: A casualty of global meltwater pulse 1A? Geology, 32(3), 249-252.

Zavala, J., De Buen, R., \& Romero, R. \&. (2011: 325). Tendencias del nivel del mar en las costas mexicanas. En A. Botella, S. Villanueva, \& J. \&. Gutiérrez, Vulnerabilidad de las zonas costeras mexicanas ante el cambio climático (Vol. I, págs. 315-333). México, Campeche, México: Universidad Autónoma de Campeche.

INAH. (2017). Catálogo de sitios arqueológicos Tampico. (I. N. Historia, Ed.) México, Ciudad de México, México.

\section{Notas}

1 Con una equidistancia de $50 \mathrm{~m}$ se construyó, una malla de $1 \mathrm{~km} 2$, en formato shape, la cual se exportó como curva a formato KML para su tratamiento en Google Earth y en 3D-Route Builder y así tabular la coordenadas "x, y, z". Con ayuda de TCX-Converter la tabla se exportó a formato CSV para poder generar una interpolación desde Quikgrid. Las isolíneas resultantes se exportaron a DXF para su tratamiento cartográfico en GvSIS. Todo el proceso se iteró más de 20 veces.

2 Cognitivamente eso equivale 800 canchas de soccer.

3 De acuerdo con INEGI (2012), el estado de Tamaulipas produjo en 2010: \$386,482.2 millones de pesos.

4 Tomando como base $\$ 400,000$ pesos por $100 \mathrm{~m} 2$, sin contar el valor de las edificaciones.

5 Como un caso análogo, tómese en cuenta que en 2005, los daños materiales causados por el huracán Katrina sobre Nuevo Orleans ascendieron a 108 mil millones de dólares, cifra equivalente al 12.89\% del PIB de México en 2010; o bien las pérdidas económicas que generó el huracán Wilma sobre Cancún ascendieron a 1,752 millones de dólares, 0.21\% del PIB nacional de 2010. 